\title{
Constraining the origin of UHECRs and astrophysical neutrinos
}

\author{
Marco Stein Muzio, ${ }^{a, *}$ Glennys R. Farrar $^{a}$ and Michael Unger ${ }^{b}$ \\ ${ }^{a}$ Center for Cosmology and Particle Physics, Department of Physics, New York University, \\ 726 Broadway, New York, New York, USA \\ ${ }^{b}$ Institut für Kernphysik, Karlsruher Institut für Technologie, \\ 76021 Karlsruhe, Germany \\ E-mail: msm659@nyu.edu, gf25@nyu.edu, Michael.Unger@kit.edu
}

\begin{abstract}
We explore the possibility of a common origin of ultrahigh energy cosmic rays (UHECRs) and astrophysical neutrinos and further constrain sources which are consistent with this possibility. We show that the common origin hypothesis can be satisfied for the SIBYLL2.3C hadronic interaction model but is difficult to accommodate for EPOS-LHC, showing that multimessenger analyses have the power to also constrain hadronic physics beyond LHC energies. We constrain properties of UHECR source environments (and potentially astrophysical neutrino sources), including their photon temperature, gas density, size, magnetic field strength and coherence length, using UHECR and neutrino spectra and composition. Our analysis represents a new type of information on UHECR sources, independent of the mechanism responsible for the UHECR acceleration.
\end{abstract}

$37^{\text {th }}$ International Cosmic Ray Conference (ICRC 2021)

July 12th - 23rd, 2021

Online - Berlin, Germany

\footnotetext{
${ }^{*}$ Presenter
} 


\section{Introduction}

As the era of multimessenger astrophysics dawns, the observation of high-energy neutrinos coincident with a flaring blazar [1] and a tidal disruption event [2] underscore the key role these particles will play in the discovery of the source of ultrahigh energy cosmic rays. The origin of ultrahigh energy cosmic rays (UHECRs, $E \gtrsim 10^{18} \mathrm{eV}=1 \mathrm{EeV}$ ) remains an open question in astroparticle physics but it is well-established that these particles will produce secondary gamma-rays and neutrinos as they propagate through the environment surrounding their source and through extragalactic space. By combining the observations of cosmic rays, gamma-rays, and neutrinos significant progress can be made in determining the sources of UHECRs, their astrophysical properties, and the relation between UHECRs and astrophysical neutrinos.

Here we employ a multimessenger analysis using the flexible, realistic phenomenological model of UHECR source environments first developed in [3] by Unger, Farrar, and Anchordoqui (UFA15). We have elaborated this model to include interactions with gas, in addition to ambient photons, in the source environment. Further, we have improved the modeling of the CR escape time to account for both the transition from diffusive to quasi-ballistic diffusion regimes and the finite size of sources. Throughout this work we consider two hadronic interaction models for both calculating secondaries from hadronic interactions in the source environment and to interpret air shower observables: EPOS-LHC [4] and SiBYLL2.3C [5].

Here we investigate the following questions:

- Can current multimessenger data distinguish gas-dominated and photon-dominated sources?

- To what extent can multimessenger data probe the acceleration mechanism producing UHECRs?

- To what extent can UHECR sources account for the flux of astrophysical neutrinos?

- What astrophysical properties (and source types) are favored by multimessenger data to explain UHECR data alone or simultaneously with some portion of the astrophysical neutrino spectrum?

We study these questions by fitting the UFA15 CR source model to UHECR spectrum and composition data from the Pierre Auger Observatory [6-12] shifted by $+20 \%$ in the energy scale and $-11 \mathrm{~g} / \mathrm{cm}^{2}$ in $\left\langle X_{\max }\right\rangle$ on average. Models are excluded based on their UHECR predictions if they produce a fit more than $2.58 \sigma$ from the best-fit, defining the number of sigma from the best-fit as $N_{\sigma}^{\prime}=S^{-1} \sqrt{\chi_{\text {model }}^{2}-\chi_{\min }^{2}}$, where $S=\sqrt{\chi_{\min }^{2} / N_{\text {dof }}}[13,14]$. In addition, we compare the model's secondary neutrino and gamma-ray flux predictions to upper-bounds from IceCube $[15,16]$ and observations from the Fermi-Large Area Telescope (LAT) [17]. Models are then excluded if they violate these upper-bounds. Specifically, we exclude models predicting more than 4.74 neutrinos above $10^{15.9} \mathrm{eV}$ (where none have yet been observed), or a gamma-ray flux greater than the measured extragalactic gamma-ray background (EGB) plus one error bar (assuming Galactic foreground model B [17]) in any given energy bin.

In order to study the ability of UHECR sources to account for the astrophysical neutrino flux, we compare the predicted neutrino flux to the IceCube Cascades data set [18] and the differential 
flux measurements from the Glashow event paper [16]. The goodness-of-fit is calculated using a $\chi^{2}$ to the data points with an additional $2 n_{i}$ penalty for energy bins with upper-bounds, where $n_{i}$ is the predicted number of events in energy bin $i$ [19]. UHECR source model parameters are not tuned to obtain the optimal goodness-of-fit to the astrophysical neutrino data. Since there is no a priori reason to assume the entire astrophysical neutrino flux should be produced by UHECR sources, we allow for a non-UHECR originating neutrino component parametrized as a single power law with an exponential cutoff. This introduces three free parameters (the power-law index, cutoff energy, and normalization) which are tuned to optimize the goodness-of-fit.

\section{Results}

A summary of our results, as were presented at the conference, follows below. For a detailed discussion of this work see [20,21].

When considering UHECR data alone, both photon- and gas-dominated source environments are able to fit the spectrum and composition. Overall though, the quality of fit is worse for gasdominated source environments - particularly when the EPOS-LHC hadronic interaction model is assumed, in which case they are mostly excluded (see Fig. 1, where $r_{\text {esc }} \equiv\left\langle N_{\text {int }}^{\text {ref }}\right\rangle$ is the average number of interactions before escape and $r_{g \gamma} \equiv\left\langle N_{\gamma}^{\mathrm{ref}}\right\rangle /\left\langle N_{g}^{\mathrm{ref}}\right\rangle$ is the ratio of the average number of photohadronic and hadronic interactions for the reference nucleus $10 \mathrm{EeV}^{56} \mathrm{Fe}$ ). Gas-dominated source environments are in tension with constraints from high-energy neutrinos, regardless of the hadronic interaction model considered. By contrast, gamma-ray data is only constraining when CRs undergo a large number of interactions on average before escaping the source environment. In all cases, we find that gamma-ray constraints on UHECR sources are weaker, and fully captured by, constraints from high-energy neutrino bounds. For this reason and for simplicity, we set aside gamma-ray constraints throughout the remainder of this proceeding.

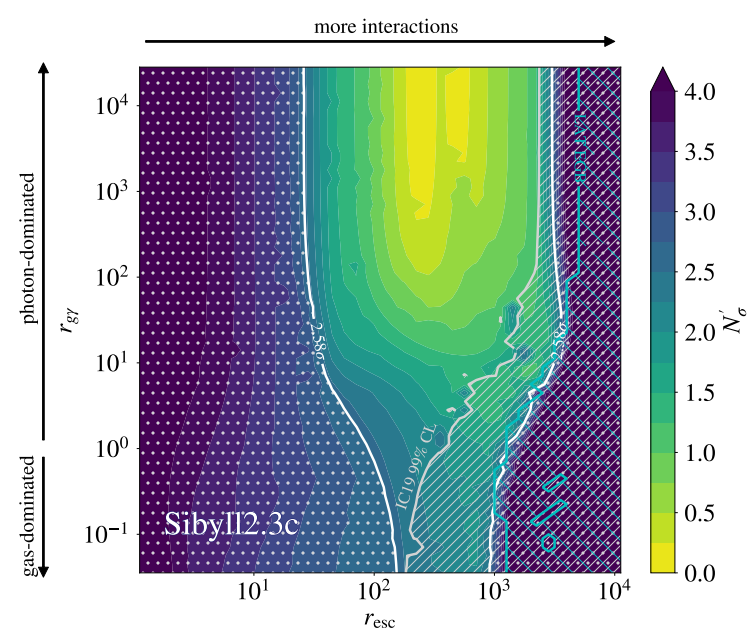

(a)

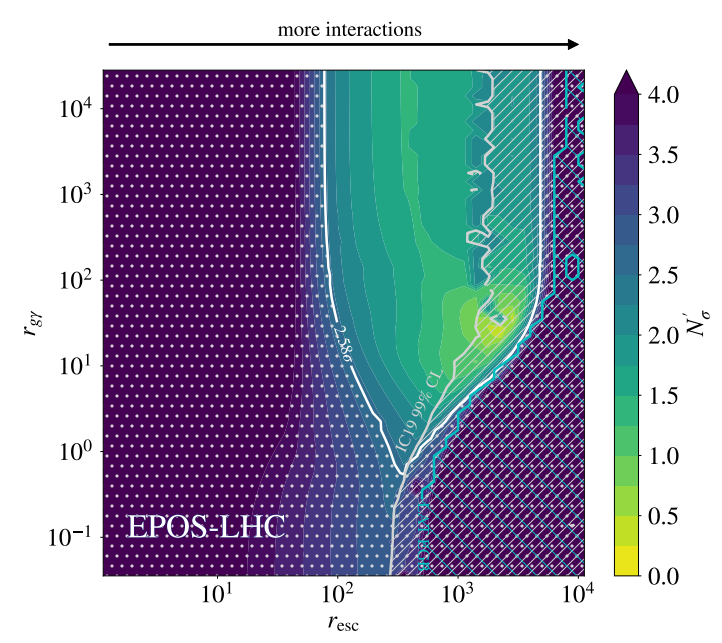

(b)

Figure 1: $N_{\sigma}^{\prime}$, the number of standard deviations the local UHECR best-fit is from the global UHECR best-fit. Contours mark 99\% CL exclusions based on UHECR data (white), non-observation of neutrinos above $10^{15.9} \mathrm{eV}$ (gray), and the upper-bound on the extragalactic gamma-ray flux (cyan). 
Our results show that gas-dominated sources can explain UHECR data when the accelerator is assumed to produce a spectrum $\propto E^{\gamma_{\text {inj }}}$ even as soft as $\gamma_{\text {inj }} \lesssim-2$ if SIBYLL2.3C is assumed (see Fig. 2). Soft spectral indices such as these are predicted for conventional acceleration processes using diffusive shock acceleration. However, such soft spectral indices are in tension with constraints on neutrinos above $10^{15.9} \mathrm{eV}$. Precise measurement of the astrophysical neutrino flux at $\sim 10 \mathrm{PeV}$ will thus probe both the acceleration mechanism of UHECR sources, the nature of UHECR interactions near the source (photohadronic or hadronic), and possibly hadronic interactions at energies above the LHC.

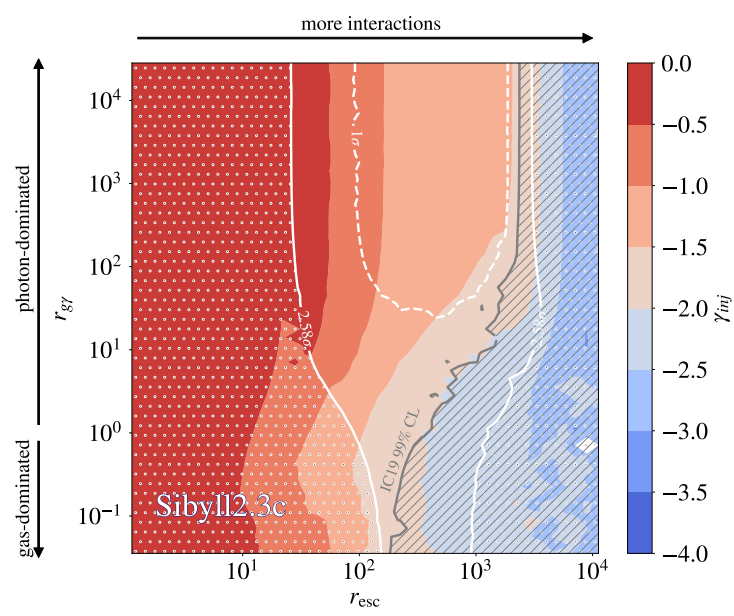

(a)

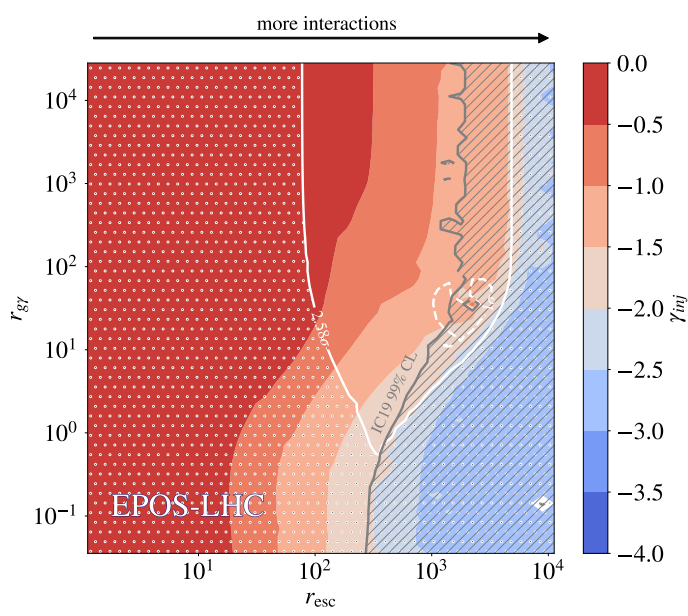

(b)

Figure 2: Spectral index $\gamma_{\text {inj }}$ of CRs injected into the source environment, $J \propto E^{\gamma_{\text {inj }}}$. Contours mark $99 \%$ CL exclusions based on UHECR data (white) and non-observation of neutrinos above $10^{15.9} \mathrm{eV}$ (gray).

We also investigated to what extent a common origin between UHECRs and astrophysical neutrinos is compatible with multimessenger data. We find that UHECR sources can explain the astrophysical neutrino flux above $\sim 1 \mathrm{PeV}$ while simultaneously giving a good fit to UHECR data and remaining compatible with gamma-ray and high-energy neutrino data (see Fig. 3). Importantly, however, it is not possible for UHECR sources to explain the entire astrophysical neutrino spectrum while remaining compatible with multimessenger data. Tantalizingly, the region of parameter space which best-fits the astrophysical neutrino flux corresponds to the best-fit region to UHECR data when assuming SiBYLL2.3c, as shown in Fig. 4a. By contrast, when assuming EPOS-LHC these regions are quite distinct, making it harder to simultaneously reconcile both the high-energy astrophysical neutrino spectrum and UHECR data.

A full Markov chain Monte Carlo exploration of the parameter space of our model was conducted to obtain preferred parameter values in order to explain UHECR data alone or simultaneously with astrophysical neutrino data - all while remaining consistent with multimessenger constraints. The parameters of our model can be mapped onto astrophysical properties of the source environment (as is shown in [21]), the preferred values for which are shown in Fig. 5a. In particular our analysis determines preferred relationship between the magnetic field strength $B$ and effective size $L$ of the source environment surrounding the accelerator. Fig. 5b shows the region of $B$ and $L$ giving the best-fit to both UHECR and astrophysical neutrino data. The approximate size and 

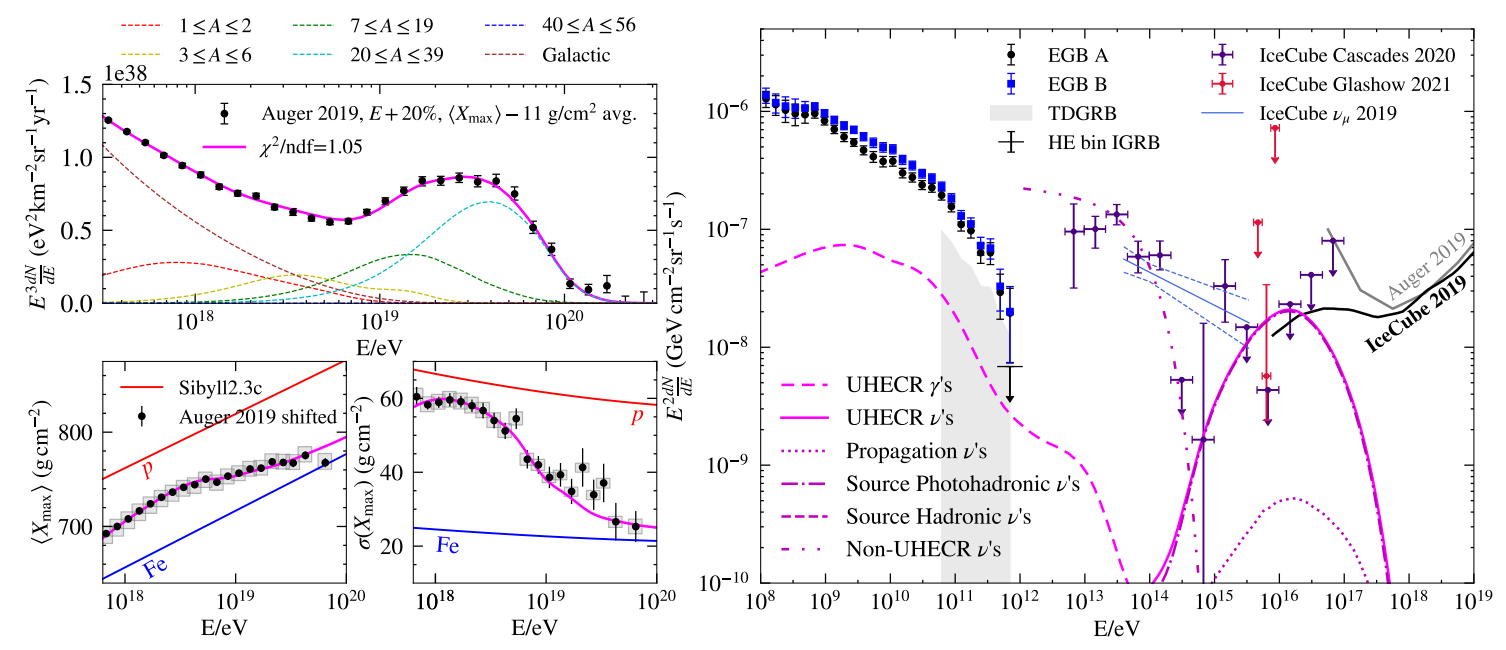

Figure 3: Predictions of the UHECR source model producing the best description of the astrophysical neutrino flux for SiBYLL2.3c. Left: The CR predictions for spectrum (top) and composition (bottom). The red and blue solid lines show the $\left\langle X_{\max }\right\rangle$ and $\sigma\left(X_{\max }\right)$ predictions of SibYLL2.3c for pure proton and iron models. Right: The neutrino and gamma-ray predictions for this model (solid and dashed lines, respectively). The neutrino flux contributions from non-UHECR sources and UHECR propagation interactions are shown separately with dot-dot-dashed, dot-dashed, and dotted dark magenta lines.

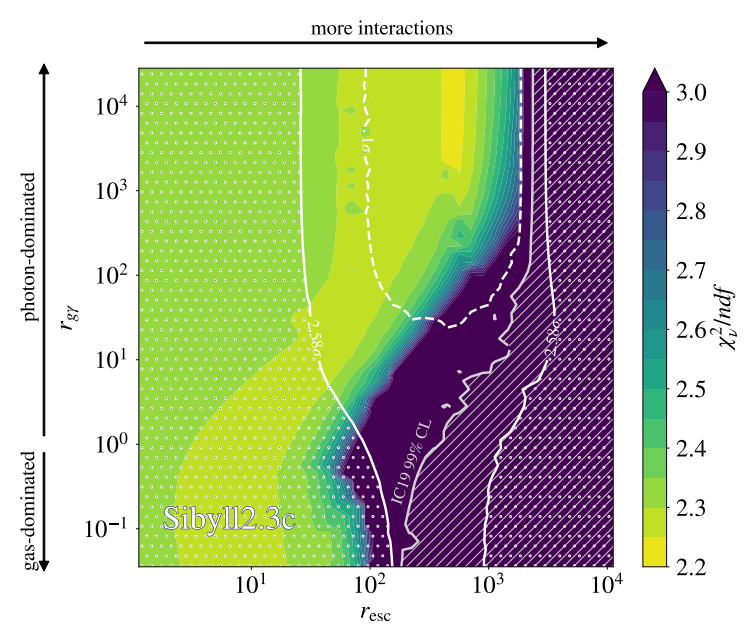

(a)

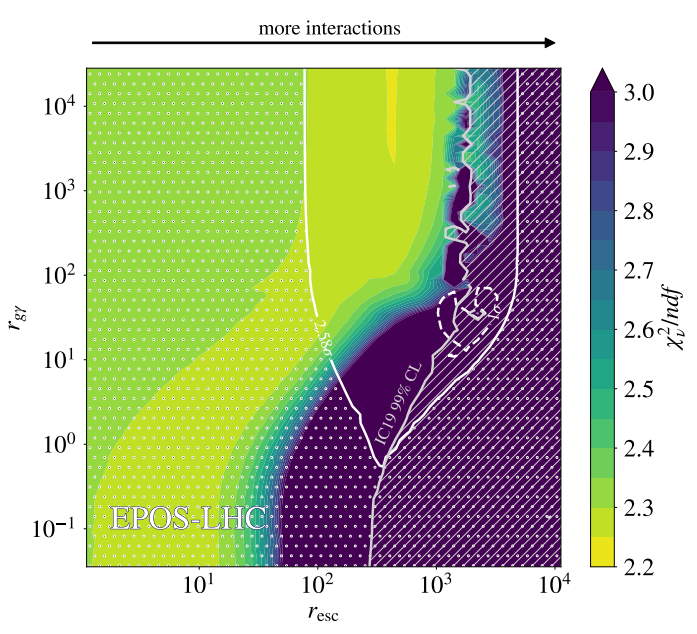

(b)

Figure 4: Reduced $\chi_{v}^{2}$ of UHECR model predictions of the neutrino flux. Contours mark best-fitting region to CR data (dashed white) and 99\% CL exclusions based on UHECR data (solid white) and non-observation of neutrinos above $10^{15.9} \mathrm{eV}$ (gray). 


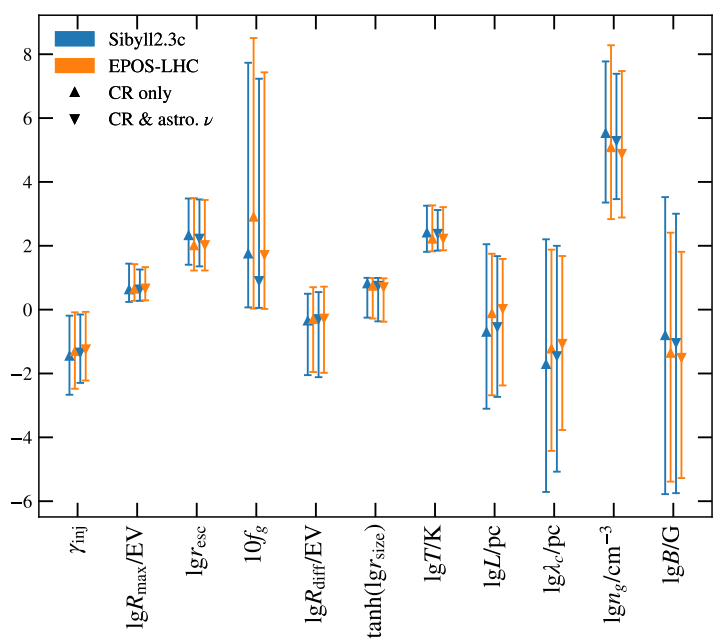

(a)

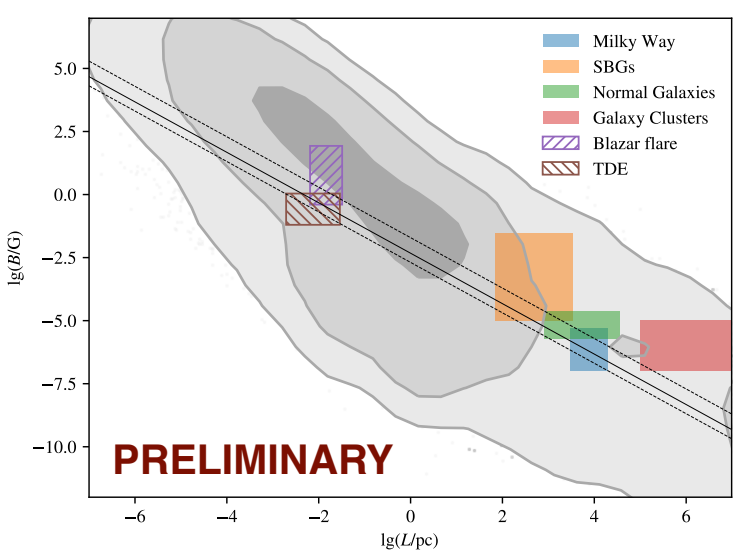

(b)

Figure 5: Left: Mode and 16th/84th percentiles of posterior distribution for various parameters fitting CR data alone (upward triangle) or simultaneously with astrophysical neutrino data (downward triangle). Right: The effective size, $L$, and magnetic field strength, $B$, of the source environment when simultaneously fitting to the UHECR spectrum and composition of Auger and the astrophysical neutrino flux of IceCube. Bands give the 1,2, and $3 \sigma$ regions (darkest to lightest grey, respectively) of the joint posterior distribution. For reference, the Hillas criterion is shown for the mode and 16th/84th percentiles (solid and dashed black lines, respectively) of the posterior distribution for $\log _{10} R_{\max }$. The indicated size and magnetic field strengths of various potential source types, taken from [2, 22-25], are approximate and serve as a guide.

magnetic field strengths of several candidate source types taken from the literature are also plotted and serve as a guide. These results, while still preliminary, suggest that smaller, $\lesssim 10 \mathrm{pc}$, source environments with stronger magnetic field strengths $\gtrsim 1 \mathrm{mG}$ are better able to explain both UHECR and astrophysical neutrino data. If so, source types like active galactic nuclei (AGN) and tidal disruption events (TDEs) appear to be favored over galaxy clusters and typical galaxies. Similar results are found when performing the MCMC analysis fitting UHECR data alone.

\section{Conclusion}

We have conducted a fully consistent multimessenger analysis of UHECR sources, their astrophysical properties, and their potential to explain the astrophysical neutrino flux. Our main findings can be summarized as follows:

- Current UHECR data can be explained by both photon- and gas-dominated source environents. However, gas-dominated environment produce a lower quality fit and are in tension with bounds on high-energy neutrinos.

- Accurate measurement of the high-energy neutrino flux, especially around $\sim 1 \mathrm{PeV}$, will be vital to determining the viability of conventional acceleration mechanisms using diffusive shock acceleration, to explain UHECR data while remaining consistent with multimessenger data. 
- UHECR sources cannot explain the entire astrophysical neutrino flux while also remaining consistent with multimessenger data. However, UHECR source can explain the flux of astrophysical neutrinos above $\sim 1 \mathrm{PeV}$, and, when assuming SiBYLL2.3C, the region of parameter space which best-fits the astrophysical neutrino flux also gives the best-fit to UHECR data.

- Small source environments, $\lesssim 10 \mathrm{pc}$, with strong magnetic fields, $\gtrsim 1 \mathrm{mG}$, are preferred by UHECR and astrophysical neutrino data and are consistent with multimessenger constraints. This favors candidate source types like AGN and TDEs over galaxy clusters and typical galaxies.

\section{References}

[1] IceCube, Fermi-LAT, MAGIC, AGILE, ASAS-SN, HAWC, H.E.S.S., INTEGRAL, Kanata, Kiso, Kapteyn, Liverpool Telescope, Subaru, Swift NuStar, VERITAS, VLA/17B-403 collaboration, Multimessenger observations of a flaring blazar coincident with high-energy neutrino IceCube-170922A, Science 361 (2018) eaat1378 [1807. 08816].

[2] R. Stein et al., A high-energy neutrino coincident with a tidal disruption event, 2005.05340.

[3] M. Unger, G. R. Farrar, and L. A. Anchordoqui, Origin of the ankle in the ultrahigh energy cosmic ray spectrum, and of the extragalactic protons below it, Phys. Rev. D 92 (2015) 123001 [1505.02153].

[4] T. Pierog et al., EPOS LHC: Test of collective hadronization with data measured at the CERN Large Hadron Collider, Phys. Rev. C92 (2015) 034906 [1306.0121].

[5] F. Riehn et al., A new version of the event generator Sibyll, PoS ICRC2015 (2016) 558 [1510.00568].

[6] Pierre Auger collaboration, Measurement of the cosmic-ray energy spectrum above $2.5 \times 10^{18} \mathrm{eV}$ using the Pierre Auger Observatory, Phys. Rev. D 102 (2020) 062005 [2008.06486].

[7] Pierre Auger collaboration, Features of the Energy Spectrum of Cosmic Rays above $2.5 \times 10^{18} \mathrm{eV}$ Using the Pierre Auger Observatory, Phys. Rev. Lett. 125 (2020) 121106 [2008.06488].

[8] Pierre Auger collaboration, Measurement of the energy spectrum of ultra-high energy cosmic rays using the Pierre Auger Observatory, PoS ICRC2019 (2020) 450.

[9] Pierre Auger collaboration, Interpretation of the Depths of Maximum of Extensive Air Showers Measured by the Pierre Auger Observatory, JCAP 1302 (2013) 026 [1301.6637].

[10] Pierre Auger collaboration, Depth of maximum of air-shower profiles at the Pierre Auger Observatory. I. Measurements at energies above $10^{17.8} \mathrm{eV}$, Phys. Rev. D 90 (2014) 122005 [1409.4809]. 
[11] Pierre Auger collaboration, Depth of maximum of air-shower profiles at the Pierre Auger Observatory. II. Composition implications, Phys. Rev. D 90 (2014) 122006 [1409. 5083].

[12] Pierre Auger collaboration, Mass Composition of Cosmic Rays with Energies above $10^{17.2}$ eV from the Hybrid Data of the Pierre Auger Observatory, PoS ICRC2019 (2020) 482.

[13] Particle Data Group collaboration, Review of Particle Physics, Chin. Phys. C 38 (2014) 090001.

[14] A. H. Rosenfeld, The particle data group: growth and operations-eighteen years of particle physics, Ann. Rev. Nucl. Part. Sci. 25 (1975) 555.

[15] ICECUBE collaboration, Differential limit on the extremely-high-energy cosmic neutrino flux in the presence of astrophysical background from nine years of IceCube data, Phys. Rev. D 98 (2018) 062003 [1807.01820].

[16] ICECuBE collaboration, Detection of a particle shower at the Glashow resonance with IceCube, Nature 591 (2021) 220.

[17] FERMI-LAT collaboration, The spectrum of isotropic diffuse gamma-ray emission between $100 \mathrm{MeV}$ and $820 \mathrm{GeV}$, Astrophys. J. 799 (2015) 86 [1410.3696].

[18] ICECUBE collaboration, Characteristics of the diffuse astrophysical electron and tau neutrino flux with six years of IceCube high energy cascade data, Phys. Rev. Lett. 125 (2020) 121104 [2001.09520].

[19] S. Baker and R. D. Cousins, Clarification of the Use of Chi Square and Likelihood Functions in Fits to Histograms, Nucl. Instrum. Meth. 221 (1984) 437.

[20] M. S. Muzio, G. R. Farrar, and M. Unger, "Ultrahigh energy cosmic rays and high energy astrophysical neutrinos." 2021 forthcoming.

[21] M. S. Muzio, G. R. Farrar, and M. Unger, "Indirect observational constraints on environments host to UHECR accelerators." 2021 in preparation.

[22] R. Jansson and G. R. Farrar, A New Model of the Galactic Magnetic Field, Astrophys. J. 757 (2012) 14 [1204.3662].

[23] T. A. Thompson, E. Quataert, E. Waxman, N. Murray, and C. L. Martin, Magnetic fields in starburst galaxies and the origin of the fir-radio correlation, Astrophys. J. 645 (2006) 186 [astro-ph/0601626].

[24] K. Ptitsyna and S. V. Troitsky, Physical conditions in potential sources of ultra-high-energy cosmic rays. I. Updated Hillas plot and radiation-loss constraints, Phys. Usp. 53 (2010) 691 [0808.0367].

[25] A. Keivani et al., A Multimessenger Picture of the Flaring Blazar TXS 0506+056: implications for High-Energy Neutrino Emission and Cosmic Ray Acceleration, Astrophys. J. 864 (2018) 84 [1807.04537]. 